UGVA-DPNC 1998/07-177 July 1998

Revised and corrected version of UGVA-DPNC 1997/10-172, CERN OPEN 97-033, hepph/9801355. To be published in Modern Physics Letters A.

\title{
INDICATIONS FOR AN ANOMALOUS RIGHT-HANDED COUPLING OF THE b-QUARK FROM A MODEL INDEPENDENT ANALYSIS OF LEP AND SLD DATA ON Z DECAYS
}

\author{
J.H.Field \\ Département de Physique Nucléaire et Corpusculaire, Université de Genève \\ 24, quai Ernest-Ansermet CH-1211Genève 4. \\ E-mail: john.field@cern.ch
}

\begin{abstract}
A model independent analysis is made using the LEP and SLD data on Z decays available at the end of 1996. The effective weak coupling constants of leptons, c quarks and b quarks are extracted. Except for the right-handed b quark coupling, they all agree with the predictions of the Standard Electroweak Model for $m_{t}=180$ $\mathrm{GeV}$ and $m_{H}=100 \mathrm{GeV}$. The right-handed b quark coupling is found to be $42 \%$ and 3.2 standard deviations above the Standard Model prediction.

PACS 13.10.+q, 13.15.Jr, 13.38.+c, 14.80.Er, 14.80.Gt

Keywords ; Standard Electroweak Model, LEP and SLD data, Z-decays, Anomalous right-handed b quark coupling.
\end{abstract}

\section{Introduction}

This letter describes a model independent analysis based on a recent compilation of LEP and SLD results on Z-decays [1]. In the first stage of the analysis the effective weak coupling constants of the charged leptons, c quarks and b quarks are extracted from the data using only weak theoretical assumptions. In the second stage a detailed comparison of the extracted effective couplings with the Standard Model (SM) [2] predictions is made. Confidence Levels (CLs) for consistency of the data with the model are calculated, taking carefully into account all important error correlations. Finally, the possible physical significance of the deviations observed from the the SM predictions for the b quark couplings is discussed. 


\section{Extraction of the Effective Weak Coupling Constants}

Instead of the vector $\left(\bar{v}_{f}\right)$ and axial vector $\left(\bar{a}_{f}\right)$ effective coupling constants for a fermion (lepton or quark) $f$, the equivalent quantities $\bar{r}_{f}$ and $\bar{s}_{f}$ are first extracted from the data. These are defined as:

$$
\begin{aligned}
& \bar{r}_{f} \equiv \bar{v}_{f} / \bar{a}_{f} \\
& \bar{s}_{f} \equiv\left(\bar{a}_{f}\right)^{2}+\left(\bar{v}_{f}\right)^{2}
\end{aligned}
$$

The experimental errors on $\bar{r}_{f}$ and $\bar{s}_{f}$ (unlike those on $\bar{v}_{f}$ and $\bar{a}_{f}$ ) are essentially uncorrelated, much simplifying the calculation of CLs for consistency with theoretical predictions. In the first comparisons with the SM shown below, the predictions are given by the global SM fit, with $m_{t}=172 \mathrm{GeV}$ and $m_{H}=149$ $\mathrm{GeV}$, from Ref.[1]. The value of the t-quark mass found in this fit is in very good agreement with the directly measured value [1] of $m_{t}=175(6) \mathrm{GeV}$ ๑. The effect of varying the Higgs boson mass, the only remaining unknown parameter of the SM, is later taken into account in the detailed comparisons with the extracted effective coupling constants.

The quantities $\bar{r}_{f}(f=\mathrm{l}, \mathrm{c}, \mathrm{b})$ and $\bar{s}_{l}$ (l is a generic lepton label) may be extracted directly from the data assuming only lepton universality. A further assumption is necessary in order to extract $\bar{s}_{c}$ or $\bar{s}_{b}$, and hence the c and b quark couplings. Since the couplings of the $\mathrm{u}, \mathrm{d}, \mathrm{s}$ quarks are only poorly measured [3], 'non-b quark lepton universality' is assumed. That is that $e$, $\mu, \tau, \mathrm{u}, \mathrm{d}, \mathrm{s}$ and $\mathrm{c}$ are all assigned the same effective weak mixing angle. Another possiblity is to assume an independently measured value of $\alpha_{s}\left(M_{Z}\right)$. The $\mathrm{c}$ and $\mathrm{b}$ quark couplings can then be extracted from $\bar{r}_{c}, \bar{r}_{b}$ and the ratios $\Gamma_{Q} / \Gamma_{l}(Q=\mathrm{c}, \mathrm{b})$, independently of the light quark effective couplings. The disadvantage of this method is that the extracted values of the heavy quark couplings are strongly correlated with the assumed value of $\alpha_{s}\left(M_{Z}\right)$.

At LEP, $\bar{r}_{f}$ is found from the measured, corrected, pole forward/backward charge asymmetries $A_{F B}^{0, f}$ [1] via the relations:

$$
\begin{aligned}
A_{F B}^{0, f} & =\frac{3}{4} A_{e} A_{f} \\
A_{f} & \equiv \frac{2 \bar{v}_{f} \bar{a}_{f}}{\left(\bar{a}_{f}\right)^{2}+\left(\bar{v}_{f}\right)^{2}}=\frac{2 \bar{r}_{f}}{1+\bar{r}_{f}^{2}}
\end{aligned}
$$

$A_{e}$ and $A_{\tau}$ have also been measured at LEP via the angular dependence of the $\tau$-polarisation asymmetry:

$$
\bar{P}_{\tau}(\cos \theta)=-\frac{A_{\tau}+A_{e} F(\theta)}{1+A_{\tau} A_{e} F(\theta)}
$$

where

$$
F(\theta) \equiv 2 \cos \theta /\left(1+\cos ^{2} \theta\right)
$$

\footnotetext{
${ }^{a}$ Throughout this letter total experimental errors ( which, unless otherwise stated, are the quadratic sum of statistical and systematic errors) are given in terms of the last significant figures. $0.1533(27)$ denotes $0.1533 \pm 0.0027$
} 
and $\theta$ is the angle between the incoming $e^{-}$and the outgoing $\tau^{-}$in the $\tau$ pair centre of mass frame. At SLD, $A_{e}$ is directly measured by the left/right beam polarisation asymmetry $A_{L R}$, while $A_{c}$ and $A_{b}$ are determined from the left/right-forward/backward asymmetries of tagged heavy quarks.

The separate LEP and SLD average values of the electroweak observables, which are directly sensitive to the effective couplings, are reported in Table 1. Also shown in Table 1 are the total errors defined as the quadratic sum of the statistical and systematic components $\left(\sigma_{T O T}=\sqrt{\sigma_{S T A T}^{2}+\sigma_{S Y S}^{2}}\right)$ as well as the systematic components $\sigma_{S Y S}$. The SM predictions for each electroweak observable and the deviations from the predictions in units of $\sigma_{T O T}$ are also reported in Table 1 . The combined LEP/SLD averages of $A_{l}, A_{c}, A_{b}, R_{c}$ and $R_{b}$, where $R_{Q}=\Gamma_{Q} / \Gamma_{\text {had }},(Q=\mathrm{c}, \mathrm{b})$ are reported in Table 2 .

The values of $\bar{r}_{f}(f=\mathrm{l}, \mathrm{c}, \mathrm{b})$ derived from the measurements of $A_{f}$, using Eqn.(4), are presented in Table 3. For the b quark, mass effects were taken into account by using the corrected form of Eqn.(4):

$$
A_{b}=\frac{2\left(\sqrt{1-4 \mu_{b}}\right) \bar{r}_{b}}{1-4 \mu_{b}+\left(1+2 \mu_{b}\right) \bar{r}_{b}^{2}}
$$

where $\mu_{b}=\left(\bar{m}_{b}\left(M_{Z}\right) / M_{Z}\right)^{2} \simeq 1.0 \times 10^{-3}$. The running b quark mass is taken as $\bar{m}_{b}\left(M_{Z}\right)=3.0 \mathrm{GeV}$ [4]. Agreement is seen with the SM at the $2 \sigma$ level for $\bar{r}_{l}$, at $<1 \sigma$ for $\bar{r}_{c}$, but only at the $3.3 \sigma$ level for $\bar{r}_{b}$. The similar discrepancy for $A_{b}$ was mentioned, but not discussed in terms of the $\mathrm{b}$ quark couplings, in Ref.[1].

It is of interest to study the sensitivity of the observed deviation of $\bar{r}_{b}$ from the SM prediction to the treatment of the experimental errors. The deviation of $3.3 \sigma$ is found when the statistical and systematic errors for each electroweak observable are added in quadrature. As estimates of systematic errors are often conservative (i.e. too high), an upper limit on the possible size of the deviation is given by neglecting all systematic errors. On the other hand, it is not clear that systematic errors should necessarily be added quadratically to statistical ones, and also the meaning of a systematic error in terms of probability content is, usually, not clearly defined. An estimate of the lower limit of the deviation is given by adding linearly the statistical and systematic errors for each electroweak observable in Table 1. Following these procedures, the following results are found:

$$
\begin{gathered}
\bar{r}_{b}=0.586(27), 3.8 \sigma \text { deviation. }\left(\sigma_{\mathrm{TOT}}=\sigma_{\mathrm{STAT}}\right) \\
\bar{r}_{b}=0.581(44), 2.5 \sigma \text { deviaton. }\left(\sigma_{\mathrm{TOT}}=\sigma_{\mathrm{STAT}}+\sigma_{\mathrm{SYS}}\right)
\end{gathered}
$$

It can be seen that the observed deviation remains at $\geq 2.5 \sigma$ independently of the treatment of systematic errors.

The quantity $\bar{s}_{l}$ is derived from the leptonic width $\Gamma_{l}$ using the relation:

$$
\bar{s}_{l}=\left(\bar{a}_{l}\right)^{2}+\left(\bar{v}_{l}\right)^{2}=\frac{12 \pi \Gamma_{l}}{\sqrt{2} G_{\mu} M_{Z}^{3}} \frac{1}{\left(1+\frac{3 \alpha\left(M_{Z}\right)}{4 \pi}\right)}
$$


The value obtained for $\bar{s}_{l}$, quoted in the first column of Table 4 , uses the LEP average value of $\Gamma_{l}$ from Table 1 together with: $G_{\mu}=1.16639 \times 10^{-5}(\mathrm{GeV})^{2}$ [ [5], $M_{Z}=91.1863 \mathrm{GeV}$, and $\alpha\left(M_{Z}\right)^{-1}=128.896$ [1]. Good agreement is found with the SM value. Solving Eqns.(1) and (2) for $\bar{a}_{l}$ and $\bar{v}_{l}$ yields the results presented in Table 5. As in the calculation of all the other effective couplings, the signs of $\bar{a}_{l}$ and $\bar{v}_{l}$ are chosen to be the same as the SM predictions. The values of $\bar{a}_{l}$ and $\bar{v}_{l}$ are in good agreement with the LEP+SLD averages quoted in Ref.[1], taking into account the slightly different analysis procedures

The quantities $\bar{s}_{Q}(Q=\mathrm{c}, \mathrm{b})$, including quark mass effects, may be derived from the measured quantities $R_{Q}$ via the relation:

$$
\bar{s}_{Q}=\left(\bar{a}_{Q}\right)^{2}\left(1-6 \mu_{Q}\right)+\left(\bar{v}_{Q}\right)^{2}=\frac{R_{Q} S_{Q}}{\left(1-R_{Q}\right) C_{Q}^{Q E D} C_{Q}^{Q C D}}
$$

where

$$
S_{Q} \equiv \sum_{q \neq Q}\left[\left(\bar{a}_{q}\right)^{2}\left(1-6 \mu_{q}\right)+\left(\bar{v}_{q}\right)^{2}\right]
$$

and [6]:

$$
\begin{aligned}
C_{Q}^{i} & =1+\delta_{Q}^{i}-<\delta_{q \neq Q}^{i}>\quad i=Q E D, Q C D ; \quad \mu_{q}=0 \text { for } q \neq b \\
\delta_{q}^{Q E D} & =\frac{3\left(e_{q}\right)^{2}}{4 \pi} \alpha\left(M_{Z}\right), \quad \delta_{q \neq b}^{Q C D}=1.00 a_{s}+1.42 a_{s}^{2}, \quad \delta_{b}^{Q C D}=.99 a_{s}-1.55 a_{s}^{2}
\end{aligned}
$$

$q$ is a generic quark flavour index, $e_{q}$ the quark electric charge in units of that of the positron and $a_{s} \equiv \alpha_{s}\left(M_{Z}\right) / \pi$. $<X>$ denotes the quark flavour average of $X$. As mentioned above, $\mu_{b}=1.0 \times 10^{-3}$ while, taking into account the present experimental error on $R_{c}, \mu_{c}$ is set to zero. The numerical values of the QED and QCD correction factors, with $\alpha_{s}\left(M_{Z}\right)=0.12$ and $\alpha\left(M_{Z}\right)^{-1}=128.9$, are presented in Table 6. The non-b quark couplings in Eqn.(8) are written, conventionally, as:

$$
\begin{aligned}
& \bar{a}_{q}=\sqrt{\rho_{q}} T_{3}^{q} \\
& \bar{v}_{q}=\sqrt{\rho_{q}}\left(T_{3}^{q}-2 e_{q}\left(\bar{s}_{W}^{q}\right)^{2}\right)
\end{aligned}
$$

where, assuming non-b quark lepton universality 0 :

$$
\begin{aligned}
\sqrt{\rho_{q}} & =\sqrt{\rho_{l}}=2\left|\bar{a}_{l}\right| \quad(\text { all } q \neq b) \\
\left(\bar{s}_{W}^{q}\right)^{2} & =\frac{1}{4}\left(1-\bar{r}_{l}\right) \quad(\text { all } q \neq b)
\end{aligned}
$$

and $T_{3}^{q}$ is the third component of the weak isospin of the quark q. Substituting the measured values of $\bar{r}_{l}, \bar{a}_{l}$, from Tables 3 and 5 and of $R_{c}, R_{b}$ from Table 2 , leads to the values of $\bar{s}_{c}, \bar{s}_{b}$ reported in Table 4 . Note that the value of $\bar{s}_{b}$, and

\footnotetext{
${ }^{b}$ Ref.[1] included small mass corrections in calculating $\bar{a}_{l}$ and $\bar{v}_{l}$ which are neglected here.

${ }^{c}$ Here the weak isospin symmetry of the SM is invoked to calculate the unmeasured couplings. It is also assumed that the quantum corrections contained in $\rho_{q}$ and $\left(\bar{s}_{W}^{q}\right)^{2}$, though not necessarily those of the SM, are universal.
} 
hence $\bar{a}_{b}$ and $\bar{v}_{b}$ are extracted first. The latter are then substituted into Eqn.(8) (taking into account their experimental errors) in order to find $\bar{s}_{c}$. In Table 4 good agreement is seen between the measured values of $\bar{s}_{l}$ and $\bar{s}_{c}$ and the SM predictions. On the other hand, $\bar{s}_{b}$ lies $1.3 \sigma$ above the prediction: a residual of the well known ' $R_{b}$ problem' [1]. Solving Eqns.(1) and (8) then gives the effective coupling constants for the heavy quarks presented in Table 7 . The values found, as well as the errors, agree well with those reported by Renton in a recent review [7]. The solutions for $\bar{a}_{f}, \bar{v}_{f}$ obtained from the essentially uncorrelated quantities $\bar{r}_{f}$ and $\bar{s}_{f}$ are shown graphically in Figs1a,1b,1c for $\mathrm{f}=l, \mathrm{c}, \mathrm{b}$ respectively. It is clear from Fig.1c that largest discrepancy with the SM is in the parameter $\bar{r}_{b}$ (completely determined by $\left.A_{b}\right)$ rather than in $\bar{s}_{b}$ ( essentially determined by $R_{b}$ ). Indeed, if the SM value for the latter is used, instead of the measured one, to solve for $\bar{a}_{f}$ and $\bar{v}_{f}$, the discrepancies between the values found and the SM are almost unchanged. The deviations
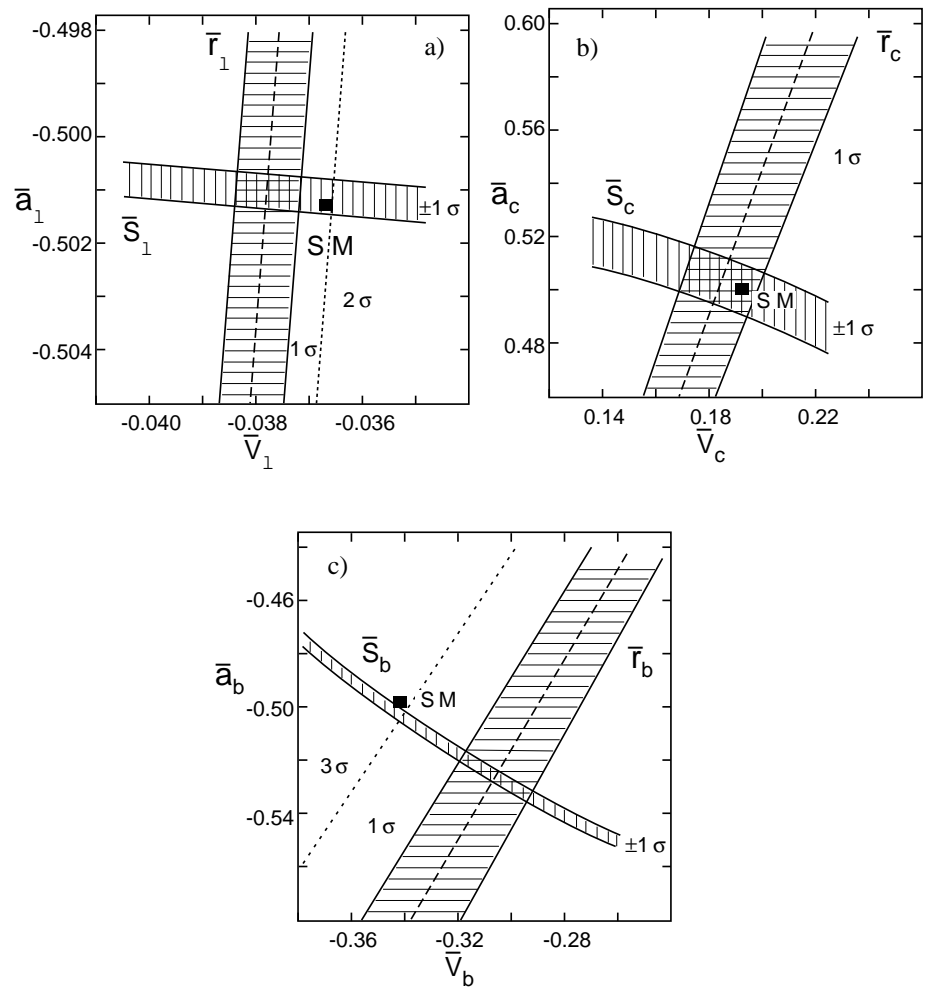

Figure 1: Constraints on the effective couplings $\bar{a}_{f}, \bar{v}_{f}$ provided by the measurements of $\bar{r}_{f}$ and $\bar{s}_{f}$. a) leptons, b) c quarks, c) b quarks. The cross-hatched areas show $\pm 1 \sigma$ limits. The dotted lines in a), $[\mathrm{c})]$ show $2 \sigma,[3 \sigma]$ limits for $\bar{r}_{l},\left[\bar{r}_{b}\right]$. SM is the Standard Model prediction for $m_{t}=172 \mathrm{GeV}, m_{H}=149 \mathrm{GeV}$.

in the b quark couplings found here are briefly compared with those that led, in 1995, to the ' $R_{b}$ problem' at the end of this letter. Although the c quark couplings agree well with the SM, and are also consistent with the quark-lepton universality hypothesis, both $\bar{a}_{b}$ and $\bar{v}_{b}$ differ from the SM values by more than three standard deviations. The errors on these quantities are, however, highly 
correlated.The statistical significance of these deviations is discussed in detail below.

It should be remarked that, although a particular value (0.12) of $\alpha_{s}\left(M_{Z}\right)$ has been assumed in order to extract the effective couplings of the heavy quarks, the sensitivity to the chosen value is very weak. Varying $\alpha_{s}\left(M_{Z}\right)$ over the range $0.1<\alpha_{s}\left(M_{Z}\right)<0.14$ leads variations of only $\simeq 3 \times 10^{-4}$ in $\bar{a}_{b}$ and $\bar{v}_{b}$ to be compared with experimental errors $\simeq 1-4 \times 10^{-2}$ (see Table 7 ).

A further constraint on the quark couplings is provided by the measurement of the mean forward/backward quark charge asymmetry:

$$
\left\langle A_{F B}^{q}\right\rangle=\frac{8 A_{l} \sum_{q} \bar{v}_{q} \bar{a}_{q}}{\sum_{q}\left[\left(1-6 \mu_{q}\right)\left(\bar{a}_{q}\right)^{2}+\left(\bar{v}_{q}\right)^{2}\right]}
$$

All experimental analyses performed to date have assumed the correctness of the SM and have used measurements of $\left\langle A_{F B}^{q}\right\rangle$ to determine a value of $\sin ^{2} \theta_{\text {eff }}^{\text {lept }}$ [1]. Inserting the average value of the latter reported in Ref.[1] into the SM formula for $\left\langle A_{F B}^{q}\right\rangle$ and propagating the error leads to the 'measured' value:

$$
\left\langle A_{F B}^{q}\right\rangle=0.1592(86)
$$

As shown in Table 8 this value is consistent with the SM prediction, with the 'model independent' prediction given by inserting the lepton and b quark couplings from Tables 5 and 7 into Eqn(13) and assuming non-b quark lepton universality for the $\mathrm{u}, \mathrm{d}, \mathrm{s}, \mathrm{c}$ quarks, as well as the prediction when, in the latter case, the measured b quark couplings are replaced by the SM ones. With the present experimental errors, $\left\langle A_{F B}^{q}\right\rangle$ is therefore insensitive to possible deviations of the $\mathrm{b}$ quark couplings from the SM, of the magnitude observed in the $A_{b}$ measurements.

As mentioned earlier, in order to avoid having to introduce an accurate value of $\alpha_{s}\left(M_{Z}\right)$ as a correlated parameter in the extraction of the heavy quark effective couplings, the hypothesis of non-b quark lepton universality was made in deriving the value of $\bar{s}_{b}$ from the measured quantity $R_{b}$. The consistency of this assumption is now checked by extracting $\alpha_{s}\left(M_{Z}\right)$ from the LEP average value of $R_{l} \equiv \Gamma_{h a d} / \Gamma_{l}$ [四]:

$$
R_{l}=20.778(29)
$$

using the relation:

$$
R_{l}=3 \frac{<C_{q}^{Q E D}><C_{q}^{Q C D}>}{C_{l}^{Q E D}} \frac{\sum_{q} \bar{s}_{q}}{\bar{s}_{l}} .
$$

The QED and QCD correction factors $<C_{q}^{Q E D}>$ and $<C_{q}^{Q C D}>$ are averaged over all quark flavours. The QED correction factors are:

$$
<C_{q}^{Q E D}>=1.00040, \quad C_{l}^{Q E D}=1.00063
$$

Inserting the measured values of $\bar{s}_{b}$ and $\bar{s}_{l}$, and using non-b quark lepton universality to evaluate $\bar{s}_{q}(\mathrm{q} \neq \mathrm{b})$ gives, for the QCD correction factor:

$$
<C_{q}^{Q C D}>=1.0394(21)
$$


Using the third order perturbative QCD formula [8]:

$$
<C_{q}^{Q C D}>=1+1.06 \frac{\alpha_{s}\left(M_{Z}\right)}{\pi}+0.9\left(\frac{\alpha_{s}\left(M_{Z}\right)}{\pi}\right)^{2}-15\left(\frac{\alpha_{s}\left(M_{Z}\right)}{\pi}\right)^{3}
$$

gives:

$$
\alpha_{s}\left(M_{Z}\right)=0.116_{-0.007}^{+0.005}
$$

which may be compared to the global fit value of Ref.[1]:

$$
\alpha_{s}\left(M_{Z}\right)=0.120(3)
$$

The good agreement of the model independent analysis result with the global world average value: $\alpha_{s}\left(M_{Z}\right)=0.118$ (5) found in two recent reviews [9, 10] of all published measurements of $\alpha_{s}$, shows that an analysis assuming this value of $\alpha_{s}\left(M_{Z}\right)$, but without the assumption of non-b quark lepton universality, would lead to essentially the same values of the b quark couplings as those reported in Table 7. In the fit used in Ref.[7] to determine the heavy quark effective couplings the constraint $\alpha_{s}\left(M_{Z}\right)=0.123(6)$ was imposed. As mentioned above, the fitted heavy quark couplings are very consistent with those found in the present analysis.

In order to correctly calculate the statistical significance of the deviations from the SM predictions of the effective couplings shown in Tables 5 and 7 it is necessary to take into account the correlations between the errors on the different quantities. To avoid the very large correlations between the errors on $\bar{a}_{f}$ and $\bar{v}_{f}$ (for the case of b quarks the correlation coefficient is -0.96) it is convenient to use, in calculating the $\chi^{2}$, the equivalent quantities $\bar{r}_{f}, \bar{s}_{f}$ for which the errors are uncorrelated for a given fermion flavour $f$. Important correlations still exist, however, between the errors on $\left(\bar{r}_{l}, \bar{r}_{c}\right)$ and $\left(\bar{r}_{l}, \bar{r}_{b}\right)$ in the case that $\bar{r}_{c}$ and $\bar{r}_{b}$ are extracted from forward/backward asymmetries using Eqns.(3),(4) and (6). The correlation coefficient is:

$$
\mathcal{C}_{l Q}=-\frac{\left(1-\bar{r}_{l}^{2}\right)\left(1+\bar{r}_{Q}^{2}\right)}{\left(1+\bar{r}_{l}^{2}\right)\left(1-\bar{r}_{Q}^{2}\right)} \frac{\sigma_{\bar{r}_{l}}}{\bar{r}_{l}} \frac{\bar{r}_{Q}}{\sigma_{\bar{r}_{Q}}}, \quad(Q=c, b)
$$

Substituting the parameters from Table 3 gives:

$$
\mathcal{C}_{l c}=-0.29, \quad \mathcal{C}_{l b}=-0.52
$$

The results on the CLs for the agreement with the SM of different sets of effective weak coupling constants, parameterised in terms of $\bar{r}_{f}$ and $\bar{s}_{f}$, are collected in Table 9. The CLs assume perfect statistical consistency of the different measurements contributing to the averages. The entries in the first column of Table 9, giving the level of agreement of $\left(\bar{r}_{l}, \bar{s}_{l}\right)$ with the SM prediction are simply calculated from the entries of Tables 3 and 4 using a diagonal error matrix, since the errors on $\bar{r}_{l}$ and $\bar{s}_{l}$ are uncorrelated. Calculating separately the contributions to $\chi^{2}$ from $\bar{r}_{l}$ and $\bar{r}_{b}$, where the latter is derived from the LEP $A_{F B}^{0, b}$ measurement, and $\bar{r}_{b}$ derived via Eqn.(6) directly from the SLD 
$A_{b}$ measurement, gives the entries reported in the second column of Table 9. The CL for agreement with the SM prediction is $1.4 \%$. The third column of Table 9 results from adding to the $\chi^{2}$ in the second column the (uncorrelated) contributions of $\bar{s}_{l}$ and $\bar{s}_{b}$. In the fourth column of Table 9 the $\chi^{2}$ and CL of the variables $\bar{r}_{l}, \bar{r}_{b}$ and $\bar{r}_{c}$ taking into account the $\bar{r}_{l}-\bar{r}_{b}$ and $\bar{r}_{l}-\bar{r}_{c}$ correlations are given. In the last column of Table 9 the (uncorrelated) variables $\bar{s}_{l}, \bar{s}_{b}$ and $\bar{s}_{c}$ are added to those of the fourth column. Note that the number of degrees of freedom corresponding to the $\chi^{2}$ values reported in the second, third, fourth and fifth columns of Table 9 are 3, 5, 5 and 8 respectively, since the $\bar{r}_{c}$ and $\bar{r}_{b}$ measurements derived from the SLD $A_{c}, A_{b}$ determinations give separate, uncorrelated, contributions to the $\chi^{2}$. As expected, the agreement with the $\mathrm{SM}$ improves as the number of degrees of freedom of the $\chi^{2}$ increases (the more parameters are considered, the more likely is a deviation associated with any of the parameters to be consistent with a statistical fluctuation).

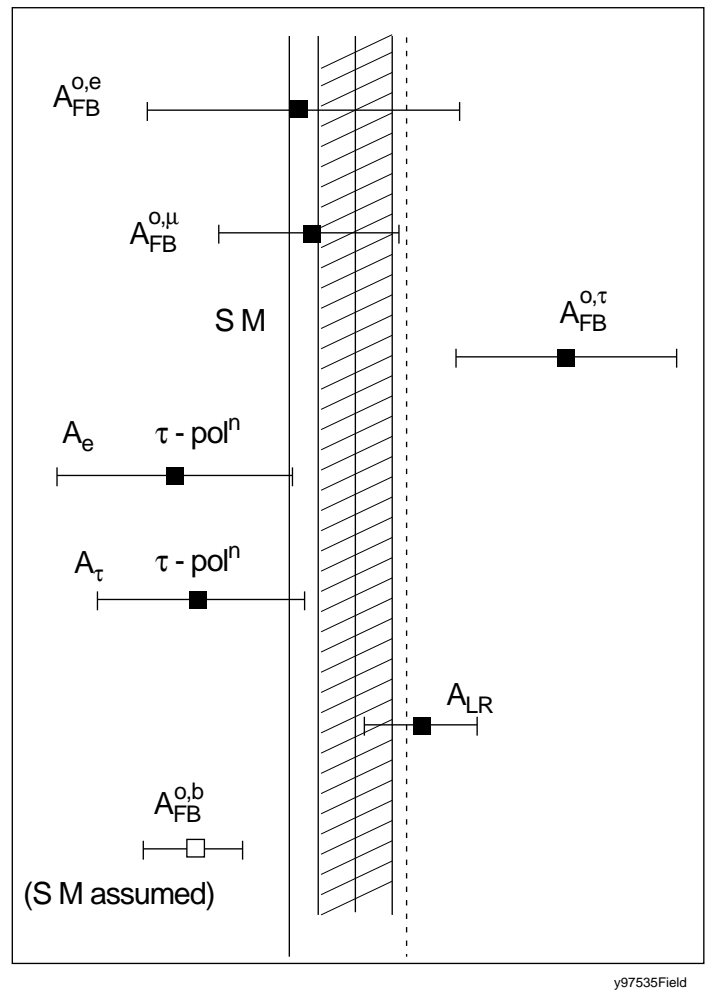

Figure 2: LEP and SLD $A_{l}$ measurements. The hatched band shows the $\pm 1 \sigma$ region around the weighted average value. The weighted average value, excluding the $\tau$ polarisation measurements, is given by the dashed line. The solid line is the Standard Model prediction for $m_{t}=172 \mathrm{GeV}, m_{H}=149 \mathrm{GeV}$. The open square shows the value of $A_{l}$ derived from the LEP average value of $A_{F B}^{0, b}$ assuming the SM; this datum is not included in the weighted averages shown.

As previously stated, the CLs shown in Table 9 are calculated on the as-

\footnotetext{
${ }^{d}$ Actually there is a weak correlation between $\bar{s}_{b}$ and $\bar{r}_{l}$ following from Eqn.(8), where $\bar{r}_{l}$ is used to calculate $S_{Q}$. However the correlation coefficient is only $\simeq 0.08$ and is neglected here.
} 
sumption that the different experiments contributing to the average values of $A_{l}, A_{c}$ and $A_{b}$ are consistent with each other. The average value of $A_{l}$ is also derived assuming lepton universality. Although the consistency of the nine different measurements of $A_{F B}^{0, b}$ [1] contributing to the LEP average value of $A_{b}$ [1] with their weighted mean value is very good $\left(\chi^{2}=5.9\right.$ for 8 dof, $\left.\mathrm{CL}=66 \%\right)$ this is not the case for the different LEP and SLD measurements of $A_{l}$ shown in Fig. 2. The overall consistency of the different measurements is only fair $\left(\chi^{2}=9.7\right.$ for $\left.5 \operatorname{dof}, \mathrm{CL}=8.4 \%\right)$ and, as discussed in detail elsewhere [1]], there are several problems of internal consistency, particularly with tau-related measurements. The dashed line in Fig. 2 shows the weighted average value of $A_{l}$ if the $\tau$-polarisation measurements are excluded. It changes by more than one standard deviation, and the internal consistency of the remaining data points is improved $\left(\chi^{2}=3.85\right.$ for 3 DOF , CL $\left.=28 \%\right)$. Repeating the analysis described above, but excluding the $\tau$-polarisation data, gives b quark couplings with even larger deviations $(\simeq 4 \sigma)$ from the SM predictions [11]. Taking into account the CL $(8.4 \%)$ for self- consistency of the different $A_{l}$ measurements, the CL that all six effective couplings are consistent with lepton universality and the SM is $0.9 \%$. The similar CL for the leptonic and b quark couplings alone is $0.18 \%$.

Also shown in Fig. 2 is the value of $A_{l}$ derived from $A_{F B}^{0, b}$, assuming the correctness of the SM. The value so obtained, 0.1396(33), differs from the weighted average of the purely leptonic measurements by $2.6 \sigma$ It is clear, from the analysis presented above, that this discrepancy is essentially due to the apparent deviations of the measured b quark effective couplings from the SM predictions. The quantity $\sin ^{2} \theta_{\text {eff }}^{\text {lept }}$ used in Ref.[1] is directly related to $A_{l}$ via Eqns.(4),(12). The poor consistency of the different $\sin ^{2} \theta_{\text {eff }}^{\text {lept }}$ determinations

in Table 19 of Ref.[1] results from the inclusion of values derived from $A_{F B}^{0, b}$ and $\left\langle A_{F B}^{q}\right\rangle$ which assume the correctness of the SM. This common origin, in the measured b quark couplings, of the poor agreement of the different $\sin ^{2} \theta_{\text {eff }}^{\text {lept }}$ determinations and the $3 \sigma$ deviation of the measured LEP-SLD average value of $A_{b}$ from the SM prediction, was not pointed out in Ref.[1].

\section{Standard Model Comparison and Physical Interpretation}

The test of the SM provided by measurements of Z decays at LEP and SLD is, essentially, that of its predictions for the quantum corrections, arising from massive virtual particle loops, to the Born level diagrams for $e^{+} e^{-} \rightarrow Z \rightarrow f \bar{f}$. The corrections may be conveniently expressed in terms of two parameters $\Delta \rho_{f}$ and $\Delta \kappa_{f}$ for each fermion flavour [12]. The parameters are given, in terms of 
the effective couplings, by the relations:

$$
\begin{aligned}
\Delta \rho_{f} & =-2\left(1-2\left|\bar{a}_{f}\right|\right) \\
\Delta \kappa_{l} & =\frac{\left(1-\bar{r}_{l}\right)}{4 s_{W}^{2}}-1 \\
\Delta \kappa_{c} & =3 \frac{\left(1-\bar{r}_{c}\right)}{8 s_{W}^{2}}-1 \\
\Delta \kappa_{b} & =3 \frac{\left(1-\bar{r}_{b}\right)}{4 s_{W}^{2}}-1
\end{aligned}
$$

Here, following the usual on-shell definition [13]:

$$
\sin ^{2} \theta_{W}=s_{W}^{2}=1-c_{W}^{2} \equiv 1-\frac{M_{W}^{2}}{M_{Z}^{2}}
$$

The SM predictions of Section 2 used the fixed values: $m_{t}=172 \mathrm{GeV}$, $m_{H}=149 \mathrm{GeV}$ found in the global fit of Ref.[1]. The effect on the SM prediction of varying $m_{t}$ and $m_{H}$ within the existing experimental bounds [1, 14 is now considered. The dependence of $\Delta \rho_{f}$ on $m_{t}$ and $m_{H}$ is contained in the terms [12]:

$$
\begin{aligned}
\Delta \rho_{f}^{\text {top }} & =\frac{3 G_{\mu} m_{t}^{2}}{8 \sqrt{2} \pi^{2}}\left(1+\xi_{f}\right) \\
\Delta \rho_{f}^{\text {Higgs }} & =-\frac{\sqrt{2} G_{\mu} M_{W}^{2}}{8 \pi^{2}} \tan ^{2} \theta_{W}\left[\frac{11}{3}\left(\ln \left(\frac{m_{H}}{M_{W}}\right)-\frac{5}{12}\right)\right]
\end{aligned}
$$

where $\xi_{f}=0$ for $f \neq b$ and $-4 / 3$ for $f=b$. The quantum correction $\Delta \kappa_{f}$ is calculated using a parameterisation of the ZFITTER [15] prediction of the effective leptonic weak mixing angle:

$$
\left(\bar{s}_{W}^{l}\right)^{2}=0.233597-8.95 \times 10^{-8} m_{t}^{2}-3.86 \times 10^{-4} \ln m_{t}+5.43 \times 10^{-4} \ln m_{H}
$$

where $m_{t}$ and $m_{H}$ are in GeV units. $\Delta \kappa_{f}$ is related to $\left(\bar{s}_{W}^{l}\right)^{2}=\left(1-\bar{r}_{l}\right) / 4$ by Eqns. (1),(9),(10),(12) and (18-20). For the b quark there is an additional non-universal contribution:

$$
\Delta \kappa_{b}^{t o p}=\frac{G_{\mu} m_{t}^{2}}{4 \sqrt{2} \pi^{2}}
$$

Although a reasonably good agreement is found for the leptonic and b quark couplings with $m_{t}=172 \mathrm{GeV}$ and $m_{H}=149 \mathrm{GeV}$ somewhat better overall agreement is found for the choice $m_{t}=180 \mathrm{GeV}$ and $m_{H}=100 \mathrm{GeV}$, still well within the current experimental bounds [1, 14]. The SM predictions for these values of $m_{t}$ and $m_{H}$ are also presented in Table 10. For b quarks however, the measured values of the quantum corrections are much larger than the SM predictions. For $\Delta \rho_{b}$ the measured value exceeds the SM prediction by a factor

${ }^{e}$ The relative accuracy of the formula $(24)$ is about one per mille for the interesting range of values of $m_{t}$ and $m_{H}$. 
of 13 , and is of opposite sign. The measured value of $\Delta \kappa_{b}$ has the same sign as the SM prediction, but is 9 times larger. Both effects are at the $>3$ standard deviation level, but they are highly correlated. The discrepancies seen are so large that the significance of the deviations is almost independent of the values of $m_{t}$ and $m_{H}$ assumed in the SM predictions.

It is also instructive to present the quantum corrections in terms of the 'epsilon parameters' introduced by Altarelli et al. [16, 17, 18]. In terms of the variables used in the present paper to describe the effective couplings, these are defined as [16]:

$$
\begin{aligned}
\epsilon_{1} & \equiv \Delta \rho_{l}=-2\left(1+2 \bar{a}_{l}\right) \\
\epsilon_{2} & \equiv c_{0}^{2} \Delta \rho_{l}+\frac{s_{0}^{2} \Delta r_{W}}{\left(c_{0}^{2}-s_{0}^{2}\right)}-2 s_{0}^{2} \Delta k^{\prime} \\
\epsilon_{3} & \equiv c_{0}^{2} \Delta \rho_{l}+\left(c_{0}^{2}-s_{0}^{2}\right) \Delta k^{\prime}
\end{aligned}
$$

here $s_{0}^{2}=1-c_{0}^{2}$ and $\Delta r_{W}$ are defined by the relations:

$$
\left(1-\frac{M_{W}^{2}}{M_{Z}^{2}}\right) \frac{M_{W}^{2}}{M_{Z}^{2}}=\frac{s_{0}^{2} c_{0}^{2}}{1-\Delta r_{W}}=\frac{\pi \alpha\left(M_{Z}\right)}{\sqrt{2} G_{\mu} M_{Z}^{2}\left(1-\Delta r_{W}\right)}
$$

and

$$
\Delta k^{\prime}=\frac{\left(1-\bar{r}_{l}\right)}{4 s_{0}^{2}}-1
$$

In Ref.[18] a fourth parameter, $\epsilon_{b}$, was introduced. It may be defined in three distinct ways:

$$
\begin{aligned}
\epsilon_{b}\left(\bar{a}_{b}\right) & \equiv \frac{\bar{a}_{b}}{\bar{a}_{l}}-1 \\
\epsilon_{b}\left(\bar{r}_{b}\right) & \equiv \frac{\bar{r}_{b}-\mathcal{R}_{l}}{1-\bar{r}_{b}} \\
\epsilon_{b}\left(\bar{s}_{b}\right) & \equiv \frac{\bar{s}_{b}-\left(\bar{a}_{l}\right)^{2}\left(1-6 \mu_{b}+\mathcal{R}_{l}^{2}\right)}{2\left(\bar{a}_{l}\right)^{2}\left(1-6 \mu_{b}+2 \mathcal{R}_{l}\right)}
\end{aligned}
$$

where

$$
\mathcal{R}_{l} \equiv \frac{\left(2+\bar{r}_{l}\right)}{3}
$$

In the SM, retaining only the leading terms $\simeq m_{t}^{2}$, the three definitions (29-31) are equivalentग. In previous phenomenological applications however, 118, 19 only the third definition (31) based, via Eqn.(8) on the measurement of $R_{b}$ was used. The measured values of the six epsilon parameters defined above are presented in Table 11, where they are compared with the SM predictions for the same values of $m_{t}$ and $m_{H}$ as in Table 10. As prevously noted 19 the values of $\epsilon_{1}, \epsilon_{2}$ and $\epsilon_{3}$ are in reasonably good agreement with the SM predictions, with deviations in the range 1-2 $\sigma$. A small deviation is also observed

\footnotetext{
${ }^{f}$ Modulo small b-mass dependent corrections
} 
for $\epsilon_{b}\left(\bar{s}_{b}\right)$, a residual of the much discussed [1] ' $R_{b}$ problem'. However, both $\epsilon_{b}\left(\bar{a}_{b}\right)$ and $\epsilon_{b}\left(\bar{r}_{b}\right)$ deviate from the SM prediction by about 4 standard deviations g. The parameter $\epsilon_{b}\left(\bar{r}_{b}\right)$ is very sensitive to the anomalous b coupling; the measured value is 39 times and $4.5 \sigma$ larger than the SM prediction. . $^{\text {The SM }}$ predictions for these quantities are very insensitive to $m_{H}$ and are dominated by the term $\simeq m_{t}^{2}$ :

$$
\epsilon_{b}=-\frac{G_{\mu} m_{t}^{2}}{4 \sqrt{2} \pi^{2}}=-0.0062 \quad\left(m_{t}=172 \mathrm{GeV}\right) .
$$

The conclusion to be drawn from Table 11 is that the deviations observed for the b quark couplings, interpreted as a real physical effect, do not enter at all into the framework of the SM nor any of its 'natural' extensions. Supersymmetry, Technicolour, anomalous $W W \gamma$ or $W W Z$ couplings, and new $U(1)$ gauge bosons are all expected, via vacuum polarisation effects in the gauge boson propagators, to produce deviations from the SM predictions for $\epsilon_{1}, \epsilon_{2}$ or $\epsilon_{3}$ [17]. No strong evidence for such deviations are observed, but rather quantum corrections to the b quark couplings that disagree, by an order of magnitude, with the expectations of the SM.

An indication of the possible physical origin of the anomalous b quark couplings is provided by considering the right- and left-handed effective couplings, $\bar{g}_{b}^{R}, \bar{g}_{b}^{L}$ related to $\bar{a}_{b}$ and $\bar{v}_{b}$ by the relations:

$$
\begin{aligned}
\bar{g}_{b}^{R}=\frac{1}{2}\left(\bar{v}_{b}-\bar{a}_{b}\right) & =-\sqrt{\rho_{b}} e_{b}\left(\bar{s}_{W}^{b}\right)^{2} \\
\bar{g}_{b}^{L}=\frac{1}{2}\left(\bar{v}_{b}+\bar{a}_{b}\right) & =\sqrt{\rho_{b}}\left[T_{b}^{3}-e_{b}\left(\bar{s}_{W}^{b}\right)^{2}\right]
\end{aligned}
$$

From the measured values of $\bar{a}_{b}$ and $\bar{v}_{b}$ presented in Table 7 , the following values of the left-handed and right-handed effective couplings of the b quarks are found:

$$
\bar{g}_{b}^{L}=-0.4155(30) \quad \bar{g}_{b}^{R}=0.1098(101)
$$

which may be compared with the SM predictions of:

$$
\bar{g}_{b}^{L}=-0.4208 \quad \bar{g}_{b}^{R}=0.0774
$$

The value of $\bar{g}_{b}^{L}$ is quite consistent with the SM prediction for $m_{t}=172 \mathrm{GeV}$, $m_{H}=149 \mathrm{GeV}$ ( a $1.3 \%, 1.8 \sigma$ deviation) whereas the discrepancy for $\bar{g}_{b}^{R}$ is much larger, (a $42 \%, 3.2 \sigma$ deviation). One may remark that the weak isospin of the SM affects only $\bar{g}_{b}^{L}$, not $\bar{g}_{b}^{R}$, so it is possible that the SM does correctly describe $\bar{g}_{b}^{L}$ but that there is a new, anomalous, right handed coupling for the b quark.

${ }^{g}$ Again, the errors on these quantities are highly correlated

${ }^{h}$ The errors on this quantity, determined essentially by those on $A_{b}$, are skewed and non-gaussian. The average error is quoted in Table 11. The confidence level of the deviation of $\epsilon_{b}\left(\bar{r}_{b}\right)$ from the SM, assuming gaussian errors for $A_{b}$, is in fact almost the same as that of the latter, about one per mille. 
The right- and left-handed effective couplings of the s,d quarks have recently been measured by the OPAL collaboration[20] with the results:

$$
\bar{g}_{d, s}^{L}=-0.44_{-0.09}^{+0.13} \quad \bar{g}_{d, s}^{R}=0.13_{-0.17}^{+0.15}
$$

to be compared with the SM predictions -0.424 and 0.077 respectively. These measurements are in good agreement with both the SM predictions and the measured b quark couplings given above.

Limits can also be set on possible anomalous couplings of the other ' $\mathrm{d}$ type' quarks, d,s by comparing the measured values of $\left\langle A_{F B}^{q}\right\rangle$ and $\Gamma_{h a d}$ with a model in which the $\mathrm{d}$ and $\mathrm{s}$ quarks are assumed to have the same effective coupling constants as those measured for the b quarks. The prediction of this model for $\left\langle A_{F B}^{q}\right\rangle$ is $0.1600(72)$, which is consistent with the 'measured' value (see Section 2 above and Table 8) of $0.1592(86)$ at the $0.68 \sigma$ level. No useful constraint on possible anomalous couplings of the $\mathrm{d}$ and $\mathrm{s}$ quarks, of a size similar to those observed for the b quark, is therefore obtained using $\left\langle A_{F B}^{q}\right\rangle$ with the present experimental errors. A more favourable case is $\Gamma_{\text {had }}$. Using the world average value of $\alpha_{s}\left(M_{Z}\right)$ of $0.118(5)$ [9, 10] in Eqn.(15) to calculate the QCD correction, and with $\left\langle C_{q}^{Q E D}\right\rangle=1.00040$, the predicted value of $\Gamma_{\text {had }}$ in the model with a universal right-handed anomaly for down-type quarks is $1.7249(46) \mathrm{GeV}$. This differs by $3.6 \sigma$ from the LEP average measurement [1] of $1.7436(25) \mathrm{GeV}$. Thus this model is essentially excluded by the measurement of $\Gamma_{\text {had }}$. It is interesting to note that the precise measurement of $\Gamma_{\text {had }}$ gives a more stringent constraint on possible anomalous couplings of the $\mathrm{d}$ and $\mathrm{s}$ quarks than the direct measurement of their left- and right-handed couplings cited above [20].

The values of the left- and right-handed couplings of the c quarks, derived from the measured values of $\bar{a}_{c}$ and $\bar{v}_{c}$ given in Table 7 , are:

$$
\bar{g}_{c}^{L}=0.3440(92) \quad \bar{g}_{c}^{R}=-0.1600(70)
$$

in very good agreement with the SM predictions for $m_{t}=172 \mathrm{GeV}, m_{H}=149$ GeV of:

$$
\bar{g}_{c}^{L}=0.3465 \quad \bar{g}_{c}^{R}=-0.1545 .
$$

The $\pm 2 \sigma$ limits for deviations of $\bar{g}_{c}^{R}$ from the SM prediction extends from 0.174 to -0.146 . Thus, at $95 \% \mathrm{CL}$, any anomalous right-handed couplings of the c quark lie between $-9 \%$ and $+15 \%$ of the SM prediction.

\section{Summary}

The confidence level that all six effective couplings extracted in the above analysis are consistent with lepton universality and the SM is found to be $0.9 \%$. This number is the product of the CL for agreement with lepton universality of the different $A_{l}$ measurements (8.4\%) shown in Fig. 2 and the CL of the SM comparison using average quantites, shown in Table 9 (10.5\%). The similarly calculated CL that the leptonic and b quark couplings alone are consistent with 
lepton universality and the SM is $0.18 \%$. The measured effective couplings of the leptons and c quarks agree well with the SM predictions, for top quark and Higgs boson masses that are consistent with the current experimental limits. In contrast to this good agreement, the extracted b quark couplings are found to deviate from the SM by more than three standard deviations. The 'epsilon parameters' extracted from the data show that such behaviour is not expected in any 'natural' extension of the SM. On the other hand, the parameter $\epsilon_{b}\left(\bar{r}_{b}\right)$ is found to be particularly sensitive to anomalous behaviour of the b quark couplings; the measured value differs from the SM prediction by $4.5 \sigma$. Comparing the left and right-handed b quark couplings with the SM predictions, the former is consistent below the $2 \sigma$ level whereas the latter shows a $42 \%$, or $3.2 \sigma$, deviation from the prediction. Thus the only significant deviation observed from the SM predictions for the effective couplings is due to the right-handed coupling of the b quark.

Finally, it may be recalled that in 1995 a difference in the world average value of $R_{b}$ from the SM prediction of about three standard deviations was reported [1]. The most recent average value is in much better agreement with the SM prediction, due to a better understanding of experimental systematics. However, as the $A_{F B}^{0, b}$ value quoted in Table 1 has a statistical error very close to the expected final LEP1 value, no large change in the average value is to be expected in the future. Of course, a hitherto unknown and correlated systematic effect in all the LEP $A_{F B}^{0, b}$ measurements cannot be excluded as the source of the apparent deviation from the SM seen in $\bar{r}_{b}$. Unfortunately, the direct measurement of $A_{b}$ at SLD is not expected to be of sufficient precision, even at the end of the experimental program, to shed much light on this possiblity. A more detailed statistical discussion of the expected future improvements in electroweak measurements, and of their probable effects on the value of $\bar{r}_{b}$, can be found in Reference 11 .

\section{Acknowledgements}

I thank M.Consoli for reading an early draft and for a useful discussion. I am also grateful to J.Mnich for providing me with results from the ZFITTER program that revealed an error in the Standard Model predictions in the previous version of this letter. I thank the referee for remarks that have improved the clarity of the presentation, and for suggesting a detailed investigation of the sensitivity of the deviations observed for the b quark couplings on the treatment of statistical and systematic errors. Finally, I should like to thank T.Junk for first making me aware of the potential problems posed, for the Standard Electroweak Model, by the measurements of the right handed b quark coupling. 


\section{References}

\section{References}

[1] The LEP Collaborations ALEPH, DELPHI, L3, OPAL, the LEP Electroweak Working Group and the SLD Heavy Flavour Group, CERNPPE/96-183 (1996).

[2] S.L.Glashow, Nucl. Phys. 22, 579 (1961). S.Weinberg Phys. Rev. Lett. 19, 1264 (1967). A.Salam in 'Elementary Particle Theory' Ed. N.Svartholm, Stockholm 1968, P367.

[3] Measurements of forward/backward asymmetries for light quarks have been made, but the current precision is rather poor. See: DELPHI Collab. P.Abreu et al. Z. Phys. C67, 1 (1995), OPAL Collab. K.Ackerstaff et al. CERN-PPE/97-063 (1997).

[4] G.Rodrigo, Nucl. Phys. B (Proc. Suppl.) 54A, 60 (1997).

[5] Particle Data Group, Phys. Rev. 54 D, 1 (1996).

[6] K.G.Chetyrkin, J.H.Kühn and A.Kwiatowski in: 'Report of the Working Group on Precision Calculations for the Z-Resonance', Eds. D.Bardin, W.Hollik and G.Passarino, CERN 95-03 (1995) P175.

[7] P.B.Renton, Int. Journ. Mod. Phys. A12, 4109 (1997).

[8] T.Hebbeker et al. Phys. Lett. B331, 165 (1994).

[9] M.Schmelling in Proceedings of the 28th International Conference on High Energy Physics, Warsaw 1996, Eds. Z.Ajduk and A.K.Wroblewski.

[10] P.N.Burrows, Talk presented at the 3rd International Symposium on Radiative Corrections, August 1-5 1996, Cracow, Poland. Pre-print: SLACPUB-7293.

[11] J.H.Field 'A Model-Independent Analysis of LEP and SLD Data on Z Decays; Is the Standard Model Confirmed?' UGVA-DPNC 1997/10-173, hep-ph/9801403. ( To be published in Phys. Rev. D)

[12] 'Z Physics at LEP1' Ed. G.Altarelli, CERN 89-08 P39,65. Notice the following misprints: In $\operatorname{Eqn}(35), \mathrm{P} 65,3 / s_{W}^{2}-2 \rightarrow 3 / s_{W}^{2}-4$ and an overall minus sign on the right side. Eqn(36), P65, 10/3 $\rightarrow$ 11/3.

[13] A.Sirlin, Phys. Rev. D22, 971 (1980), W.J.Marciano and A.Sirlin, Phys. Rev. D22, 2695 (1980); D29, 75 (1984).

[14] ALEPH Collab., R.Barate et al., Phys. Lett. B412, 173 (1997).

DELPHI Collab., B.Abreu et al., Eur. Phys. J C2, 1 (1998).

L3 Collab., M.Acciarri et al., Phys. Lett. B411, 373 (1997).

OPAL Collab. K.Ackerstaff et al., Eur. Phys. J C1, 425 (1998).

[15] D.Bardin et al. FORTRAN package ZFITTER, Preprint CERN-TH $6443 / 92$.

[16] G.Altarelli and R.Barbieri, Phys. Lett. B253, 161 (1991). 
[17] G.Altarelli, R.Barbieri and S.Jadach, Nucl. Phys. B376, 3 (1992).

[18] G.Altarelli, R.Barbieri and F.Caravaglios, Nucl. Phys. B405, 3 (1993).

[19] G.Altarelli, 'Status of Precision Tests of the Standard Model', CERNTH/91-265, October 1996.

[20] See the second reference in [3]. 


\begin{tabular}{|c|c|c|c|}
\hline Quantity & Value $\left(\sigma_{T O T}\right)\left[\sigma_{S Y S}\right]$ & SM & $($ Meas.-SM $) / \sigma_{T O T}$ \\
\cline { 1 - 3 } LEP & & & \\
\cline { 1 - 2 }$A_{F B}^{0, e}$ & $0.0160(24)[16]$ & 0.0159 & 0.04 \\
$A_{F B}^{0, \mu}$ & $0.0162(13)[5]$ & 0.0159 & 0.04 \\
$A_{F B}^{0, \tau}$ & $0.0201(18)[10]$ & 0.0159 & 2.3 \\
$\Gamma_{l}$ (Mev) & $83.91(11)[8]$ & 83.96 & -0.45 \\
$\tau$-polarisation & & & \\
$A_{e}$ & $0.1382(76)[21]$ & 0.1458 & -1.0 \\
$A_{\tau}$ & $0.1401(67)[45]$ & 0.1458 & -0.9 \\
c and b quarks & & & \\
$A_{F B}^{0, c}$ & $0.0733(49)[26]$ & 0.0730 & 0.1 \\
$R_{c}$ & $0.1715(56)[42]$ & 0.1723 & -0.1 \\
$A_{F B}^{0, b}$ & $0.0979(23)[10]$ & 0.1022 & -1.8 \\
$R_{b}$ & $0.2179(12)[9]$ & 0.2158 & 1.8 \\
\hline SLD & & & \\
\cline { 1 - 2 }$A_{e}$ & $0.1543(37)[14]$ & 0.1458 & 2.3 \\
$A_{c}$ & $0.625(84)[41]$ & 0.667 & -0.5 \\
$A_{b}$ & $0.863(49)[32]$ & 0.935 & -1.4 \\
$R_{b}$ & $0.2149(38)[21]$ & 0.2158 & -0.2 \\
\hline
\end{tabular}

Table 1: Average values of electroweak observables used in the analysis [1]. SM denotes the Standard Model prediction for $m_{t}=172 \mathrm{GeV}, m_{H}=149 \mathrm{GeV}$ [1]. $\sigma_{\text {TOT }}$ is the total experimental error, which is the quadratic sum of the statistical $\left(\sigma_{S T A T}\right)$ and systematic $\left(\sigma_{S Y S}\right)$ errors.

\begin{tabular}{|c|c|c|c|c|}
\hline$A_{l}$ & $A_{c}$ & $A_{b}$ & $R_{c}$ & $R_{b}$ \\
\hline $0.1501(24)$ & $0.645(39)$ & $0.869(22)$ & $0.1715(56)$ & $0.2176(11)$ \\
\hline
\end{tabular}

Table 2: LEP+SLD averages

\begin{tabular}{|c|c|c|c|}
\hline & $\bar{r}_{l}$ & $\bar{r}_{c}$ & $\bar{r}_{b}$ \\
\hline Measurement & $0.07548(120)$ & $0.366(29)$ & $0.582(32)$ \\
SM & 0.07332 & 0.383 & 0.689 \\
$($ Meas.-SM)/Error & -1.80 & -0.59 & -3.34 \\
\hline
\end{tabular}

Table 3: $\quad$ Measured values of $\bar{r}_{f}=\bar{v}_{f} / \bar{a}_{f}$ compared to Standard Model predictions 


\begin{tabular}{|c|c|c|c|}
\hline & $\bar{s}_{l}$ & $\bar{s}_{c}$ & $\bar{s}_{b}$ \\
\hline Measurement & $0.25244(33)$ & $0.2877(95)$ & $0.3676(24)$ \\
SM & 0.25259 & 0.2880 & 0.3644 \\
(Meas.-SM)/Error & -0.45 & -0.03 & 1.33 \\
\hline
\end{tabular}

Table 4: $\quad$ Measured values of $\bar{s}_{f}=\bar{a}_{f}^{2}\left(1-6 \mu_{f}\right)+\bar{v}_{f}^{2}$ compared to Standard Model predictions

\begin{tabular}{|c|c|c|c|}
\hline \multirow{2}{*}{} & \multicolumn{3}{|c|}{ leptons } \\
\cline { 2 - 4 } & Meas. & SM & $\operatorname{Dev}(\sigma)$ \\
\hline $\bar{a}_{f}$ & $-0.50101(33)$ & -0.50124 & 0.67 \\
$\bar{v}_{f}$ & $-0.03782(68)$ & -0.03675 & -1.57 \\
\hline
\end{tabular}

Table 5: Measured values of the effective electroweak coupling constants for the charged leptons. $\operatorname{Dev}(\sigma)=($ Meas.-SM) $/$ Error.

\begin{tabular}{|c|c|c|c|}
\hline$C_{c}^{Q E D}$ & $C_{b}^{Q E D}$ & $C_{c}^{Q C D}$ & $C_{b}^{Q C D}$ \\
\hline 1.00046 & 0.99975 & 1.0002 & 0.9953 \\
\hline
\end{tabular}

Table 6: QED and QCD correction factors for heavy quarks assuming $\alpha_{s}\left(M_{Z}\right)=0.12$ and $\alpha\left(M_{Z}\right)^{-1}=128.9$.

\begin{tabular}{|c|c|c|c|c|c|c|}
\hline \multirow{2}{*}{} & \multicolumn{3}{|c|}{ c quark } & \multicolumn{3}{c|}{ b quark } \\
\cline { 2 - 7 } & Meas. & SM & $\operatorname{Dev}(\sigma)$ & Meas. & SM & $\operatorname{Dev}(\sigma)$ \\
\hline $\bar{a}_{f}$ & $0.504(10)$ & 0.501 & 0.30 & $-0.5252(75)$ & -0.4981 & -3.61 \\
$\bar{v}_{f}$ & $0.184(15)$ & 0.192 & -0.53 & $-0.3057(125)$ & -0.3434 & 3.18 \\
\hline
\end{tabular}

Table 7: Measured values of the effective electroweak coupling constants of $\mathrm{c}$ and b quarks. $\operatorname{Dev}(\sigma)=($ Meas.-SM)/Error.

\begin{tabular}{|c|c|c|c|c|}
\hline & 'Measured' & SM Pred. & MI Pred. & MI Pred. with SM b quark \\
\hline$\left\langle A_{F B}^{q}\right\rangle$ & $0.1592(86)$ & 0.1641 & $0.1639(28)$ & $0.1692(28)$ \\
\hline ('Meas'.-Pred.)/Error & & -0.57 & -0.52 & -1.1 \\
\hline
\end{tabular}

Table 8: Values of the mean quark charge asymmetry. 'MI Pred.' stands for Model Independent Prediction (see text). See also the text for the definition of 'Measured'. 


\begin{tabular}{|c|c|c|c|c|c|}
\hline Observables & $\bar{r}_{l}, \bar{s}_{l}$ & $\bar{r}_{l}, \bar{r}_{b}$ & $\bar{r}_{l}, \bar{s}_{l}, \bar{r}_{b}, \bar{s}_{b}$ & $\bar{r}_{l}, \bar{r}_{b}, \bar{r}_{c}$ & $\bar{r}_{l}, \bar{s}_{l}, \bar{r}_{b}, \bar{s}_{b}, \bar{r}_{c}, \bar{s}_{c}$ \\
\hline$d o f$ & 2 & 3 & 5 & 5 & 8 \\
$\chi^{2}$ & 3.44 & 10.6 & 13.2 & 10.9 & 13.2 \\
CL $(\%)$ & 17.9 & 1.4 & 2.2 & 5.3 & 10.5 \\
\hline
\end{tabular}

Table 9: $\quad \chi^{2}$ and confidence levels for agreement with the SM $\left(m_{t}=172 \mathrm{GeV}, m_{H}=\right.$ $149 \mathrm{GeV}$ ) of different sets of electroweak observables sensitive to the effective couplings, assuming perfect statistical consistency of the LEP+SLD averages in Table 2. See the text for the explanation of the number of degrees of freedom $(d o f)$ in each case.

\begin{tabular}{|c|c|c|c|c|c|c|}
\hline & \multicolumn{2}{|c|}{ leptons } & \multicolumn{2}{c|}{ c quark } & \multicolumn{2}{c|}{ b quark } \\
\cline { 2 - 7 } & $\Delta \rho_{l}$ & $\Delta \kappa_{l}$ & $\Delta \rho_{c}$ & $\Delta \kappa_{c}$ & $\Delta \rho_{b}$ & $\Delta \kappa_{b}$ \\
\hline Expt. & $0.00404(133)$ & $0.03445(134)$ & $0.016(41)$ & $0.064(49)$ & $0.101(32)$ & $0.403(107)$ \\
\hline $\mathrm{SM} m_{t}=172 \mathrm{GeV}$ & & & & & & \\
$m_{H}=149 \mathrm{GeV}$ & 0.00497 & 0.03686 & 0.005 & 0.037 & -0.007 & 0.0436 \\
$\operatorname{Dev}(\sigma)$ & -0.7 & -1.8 & 0.27 & 0.55 & 3.38 & 3.36 \\
\hline $\mathrm{SM} m_{t}=180 \mathrm{GeV}$ & & & & & & \\
$m_{H}=100 \mathrm{GeV}$ & 0.00563 & 0.03472 & 0.006 & 0.034 & -0.008 & 0.0412 \\
$\operatorname{Dev}(\sigma)$ & -1.2 & -0.02 & 0.24 & 0.61 & 3.40 & 3.38 \\
\hline
\end{tabular}

Table 10: Measured values of the quantum correction parameters $\Delta \rho_{f}$ and $\Delta \kappa_{f}$ compared to $\mathrm{SM}$ predictions. $\operatorname{Dev}(\sigma)=($ Expt.-SM)/Error.

\begin{tabular}{|c|c|c|c|c|c|c|}
\hline & $\epsilon_{1}$ & $\epsilon_{2}$ & $\epsilon_{3}$ & $\epsilon_{b}\left(\bar{s}_{b}\right)$ & $\epsilon_{b}\left(\bar{a}_{b}\right)$ & $\epsilon_{b}\left(\bar{r}_{b}\right)$ \\
\hline Expt. & $0.00404(133)$ & $-0.0073(8)$ & $0.0031(8)$ & $-0.0017(18)$ & $0.048(15)$ & $-0.263(57)$ \\
\hline $\mathrm{SM} m_{t}=172 \mathrm{GeV}$ & & & & & & \\
$m_{H}=149 \mathrm{GeV}$ & 0.00497 & -0.0076 & 0.0051 & -0.0045 & -0.0060 & -0.0068 \\
$\operatorname{Dev}(\sigma)$ & -0.7 & 0.38 & -2.5 & 1.6 & 3.6 & -4.5 \\
\hline $\mathrm{SM} m_{t}=180 \mathrm{GeV}$ & & & & & & \\
$m_{H}=100 \mathrm{GeV}$ & 0.00563 & -0.0062 & 0.0045 & -0.0046 & -0.0068 & -0.0055 \\
$\operatorname{Dev}(\sigma)$ & -1.2 & -1.4 & -1.8 & 1.6 & 3.7 & -4.5 \\
\hline
\end{tabular}

Table 11: Measured values of the epsilon parameters of Refs.[16-18] compared to SM predictions. $\operatorname{Dev}(\sigma)=($ Expt.-SM)/Error. 